\title{
Probing long-lived particles at Higgs factories
}

\author{
Kingman Cheung ${ }^{1,2,3, *}$ and Zeren Simon Wang $\odot^{4,1, \dagger}$ \\ ${ }^{1}$ Physics Division, National Center for Theoretical Sciences, Hsinchu 300, Taiwan \\ ${ }^{2}$ Department of Physics, National Tsing Hua University, Hsinchu 300, Taiwan \\ ${ }^{3}$ Division of Quantum Phases and Devices, School of Physics, Konkuk University, \\ Seoul 143-701, Republic of Korea \\ ${ }^{4}$ Asia Pacific Center for Theoretical Physics (APCTP)—Headquarters San 31, \\ Hyoja-dong, Nam-gu, Pohang 790-784, Korea
}

(Received 11 December 2019; accepted 22 January 2020; published 5 February 2020)

\begin{abstract}
We study displaced vertex signatures of long-lived particles (LLPs) from exotic Higgs decays in the context of a Higgs-portal model and a neutral-naturalness model at the circular electron positron collider (CEPC) and future circular collider $e^{+} e^{-}$(FCC-ee). Such two models feature two representative mass ranges for LLPs, which show very different behavior in their decay signatures. The Higgs-portal model contains a very light sub-GeV scalar boson stemming from a singlet scalar field appended to the Standard Model. Such a light scalar LLP decays into a pair of muons or pions, giving rise to a distinctive signature of collimated muon-jet or pion-jet, thanks to the sub-GeV mass. On the other hand, the neutral-naturalness model, e.g., folded supersymmetry, predicts the lightest mirror glueball of mass $O(10) \mathrm{GeV}$, giving rise to long decays with a large transverse impact parameter because of the relatively large mass. Utilizing such distinct characteristics to remove the background, we estimate the sensitivities of searches for light scalar bosons and mirror glueballs at the CEPC and FCC-ee. We find either complementary or stronger coverage compared to the previous results in the similar contexts.
\end{abstract}

DOI: 10.1103/PhysRevD.101.035003

\section{INTRODUCTION}

Since the discovery of a Standard Model (SM)-like Higgs boson at the LHC in 2012 [1,2], there has been no sign of new physics in collider experiments so far. As most effort at high-energy colliders, e.g., the LHC has been paid for searching for promptly decaying new heavy fields, new physics that would emerge in the form of long-lived particles (LLPs) predicted in various models beyond the Standard Model (BSM) might have been missed, as the current triggers for events are not designed specifically for such purposes. In fact, the rising interest in searches for LLPs has injected life into both theoretical and experimental communities [3]. Many BSM models have been proposed that predict existence of LLPs, either charged or neutral: Higgs-portal models, $R$-parity violating (RPV) supersymmetry with small RPV couplings, quirky models, gauge-mediated models, etc. For a summary, see Ref. [3]. Some searches for LLPs have also been performed

\footnotetext{
cheung@phys.nthu.edu.tw

zerensimon.wang@apctp.org
}

Published by the American Physical Society under the terms of the Creative Commons Attribution 4.0 International license. Further distribution of this work must maintain attribution to the author(s) and the published article's title, journal citation, and DOI. Funded by SCOAP ${ }^{3}$. at ATLAS and CMS: see, for example, Refs. [4-8]. Nevertheless, the current triggers are not optimized for detecting LLPs so that perhaps many signals might have been missed. Specific triggers will be installed in future runs at the ATLAS and CMS experiments $[9,10]$.

Other than the high-luminosity run at the LHC, there are also proposals for the next generation $e^{-} e^{+}$colliders, such as circular electron positron collider (CEPC) [11], future circular collider $e^{+} e^{-}$(FCC-ee) [12], and ILC [13]. These future colliders, if they can be approved, all involve an important phase of operation as a Higgs factory running at center-of-mass energies $\sqrt{s}=240-250 \mathrm{GeV}$. In such machines, the Higgs bosons are dominantly produced by Higgsstrahlung together with a relatively small contribution from $W W / Z Z$ fusion. Producing the Higgs bosons copiously with relatively little background, such Higgs factories are expected to be ideal avenues for precision measurements of the Higgs couplings and other properties of the Higgs boson, including searching for rare decays of the Higgs boson. One particularly interesting rare decay possibility is the Higgs decay into LLPs. Though charged LLPs could be easily identified as stable charged tracks in tracker detectors at the LHC, neutral LLPs would be easily missed in the current searches.

In this work, we study the sensitivity reach of the CEPC and FCC-ee for some neutral LLPs produced from rare 
Higgs decays. We use a Higgs-portal model and a dark glueball model as the prototype models. These models share the same features that the neutral LLPs are scalar bosons pair produced from the SM Higgs decays and followed by their predominant decays into a pair of leptonic or hadronic jets. We first investigate the dimuon decay of the new scalar bosons of the Higgs-portal model. It has the advantage that the muons can be detected quite cleanly in both the inner tracker (IT) detector and muon spectrometer (MS). The detection and identification efficiencies are in general very high. The new scalar boson of the toy models mentioned above has distinct interesting mass ranges. The Higgs-portal model [14] that we are interested in allows the new scalar boson as light as sub-GeV. Because of the light mass, it will be traveling with a high transverse momentum in the Higgs decay, such that the opening angle between the dimuons would be order $\Delta R_{\mu \mu} \sim 2 m_{h_{s}} / p_{T}=O\left(10^{-2}\right)$. Making use of this feature one can effectively eliminate the background events. The light scalar boson could as well decay into a pair of (charged) pions with the same feature as the collimated jets. We also take into account this possibility by considering reconstructing the displaced vertices in the IT, HCAL, or MS. On the other hand, the dark glueball lies in the mass range of a few tens of GeV. We focus on the mirror glueball that decays to a pair of $b$ jets, given the fact that the decay branching ratios of the mirror glueball follow the pattern of the SM-like Higgs boson of the same mass. Given the relatively large mass of the mirror glueball, the $b$-jet pair will have a wide opening angle. These two models thus provide two distinct representatives in the search of such LLPs.

The organization of this paper is as follows. In the next section, we highlight on the two representative models studied in this work. In Sec. III, we describe briefly the layout of the CEPC and FCC-ee detectors, and detail our search strategies and simulation procedures. We present the results in Sec. IV and conclude in Sec. V.

\section{TWO MODELS FOR THE LONG-LIVED PARTICLES}

\section{A. A Higgs-portal model}

In this work, we consider a toy Higgs-portal model where an additional real SM-singlet scalar field $X$ is added to the SM Lagrangian, and the field $X$ mixes with the SM Higgs doublet field $\Phi$, in the presence of a new $Z_{2}$ symmetry. The new scalar field $X$ is odd under the $Z_{2}$ such that no $X$ or $X^{3}$ terms appear, while all the SM fields are even. The renormalizable Lagrangian is given by

$$
\begin{aligned}
\mathcal{L}= & \frac{1}{2} \partial_{\mu} X \partial^{\mu} X+\frac{1}{2} \mu_{X}^{2} X^{2}-\frac{1}{4} \lambda_{X} X^{4}-\frac{1}{2} \lambda_{\Phi X}\left(\Phi^{\dagger} \Phi\right) X^{2} \\
& +\mathcal{L}_{\mathcal{S M}},
\end{aligned}
$$

where the SM Higgs sector is expressed with

$$
\mathcal{L}_{\mathcal{S M}} \supset\left(D_{\mu} \Phi\right)^{\dagger}\left(D^{\mu} \Phi\right)+\mu^{2}\left(\Phi^{\dagger} \Phi\right)-\lambda\left(\Phi^{\dagger} \Phi\right)^{2} .
$$

After the electroweak symmetry breaking, both the SM Higgs doublet field $\Phi$ and the new scalar singlet field $X$ are expanded around their vacuum-expectation values $\langle\phi\rangle \approx$ $246 \mathrm{GeV}$ and $\langle\chi\rangle$,

$$
\begin{gathered}
\Phi(x)=\frac{1}{\sqrt{2}}\left(\begin{array}{c}
0 \\
\langle\phi\rangle+\phi(x)
\end{array}\right) \\
X(x)=\langle\chi\rangle+\chi(x) .
\end{gathered}
$$

We may express the two tadpole conditions by imposing $\partial V / \partial \phi=0$ and $\partial V / \partial \chi=0$, with $V$ labeling the scalar potential part of Eq. (1),

$$
\begin{aligned}
\langle\phi\rangle^{2} & =\frac{4 \lambda_{X} \mu^{2}-2 \lambda_{\Phi X} \mu_{X}^{2}}{4 \lambda \lambda_{X}-\lambda_{\Phi X}^{2}}, \\
\langle\chi\rangle^{2} & =\frac{4 \lambda \mu_{X}^{2}-2 \lambda_{\Phi X} \mu^{2}}{4 \lambda \lambda_{X}-\lambda_{\Phi X}^{2}} .
\end{aligned}
$$

Note that if we take the decoupling limit $\lambda_{\Phi X} \rightarrow 0$ from the above equations, we can reproduce the SM condition of $\langle\phi\rangle^{2}=\mu^{2} / \lambda$ and $\langle\chi\rangle^{2}=\mu_{X}^{2} / \lambda_{X}$.

One can easily see from Eq. (1) that the two scalar fields in the model, i.e., $\phi$ and $\chi$ will mix with each other and form new mass eigenstates which we label with $h$ and $h_{s}$, respectively. The mass terms of the two scalar bosons are

$$
\mathcal{L}_{m}=-\frac{1}{2}(\phi \chi)\left(\begin{array}{cc}
2 \lambda\langle\phi\rangle^{2} & \lambda_{\Phi X}\langle\phi\rangle\langle\chi\rangle \\
\lambda_{\Phi X}\langle\phi\rangle\langle\chi\rangle & 2 \lambda_{X}\langle\chi\rangle^{2}
\end{array}\right)\left(\begin{array}{l}
\phi \\
\chi
\end{array}\right) .
$$

It is possible to rotate $(\phi \chi)^{T}$ to $\left(h h_{s}\right)^{T}$ through an angle $\theta$,

$$
\left(\begin{array}{l}
h \\
h_{s}
\end{array}\right)=\left(\begin{array}{cc}
\cos \theta & \sin \theta \\
-\sin \theta & \cos \theta
\end{array}\right)\left(\begin{array}{l}
\phi \\
\chi
\end{array}\right) .
$$

The angle $\theta$ has to be small because of various existing constraints [14], so we will focus on small $\theta$ values for the rest of this section. As a result, we may express the masses of $h$ and $h_{s}$, the mixing angle $\theta$, and the interaction term for a three-point vertex $h h_{s} h_{s}$ in terms of the parameters in Eq. (1) as

$$
\begin{aligned}
m_{h}^{2} & \simeq 2 \lambda\langle\phi\rangle^{2}=(125.10 \mathrm{GeV})^{2} \\
m_{h_{s}}^{2} & \simeq 2 \lambda_{X}\langle\chi\rangle^{2} \\
\mathcal{L}_{h h_{s} h_{s}} & =-\frac{1}{2} \lambda_{\Phi X}\langle\phi\rangle h h_{s} h_{s} \\
\theta & \simeq \frac{\lambda_{\Phi X}\langle\phi\rangle\langle\chi\rangle}{m_{h}^{2}-m_{h_{s}}^{2}} .
\end{aligned}
$$

Because of its mixing with the Higgs boson, the scalar boson $h_{s}$ can decay into SM particles with the decay rate 
TABLE I. The decay branching ratios for the two most dominant decay modes of the scalar boson $h_{s}$ for $m_{h_{s}}=0.3-1 \mathrm{GeV}$. Here, $\pi \pi$ includes $\pi^{+} \pi^{-}$and $\pi^{0} \pi^{0}$.

\begin{tabular}{lcccccccc}
\hline \hline$m_{h_{s}}(\mathrm{GeV})$ & 0.3 & 0.4 & 0.5 & 0.6 & 0.7 & 0.8 & 0.9 & 1.0 \\
\hline $\operatorname{Br}\left(\mu^{+} \mu^{-}\right)$ & $20.6 \%$ & $13.0 \%$ & $10.3 \%$ & $8.6 \%$ & $7.1 \%$ & $5.1 \%$ & $2.5 \%$ & $2.0 \%$ \\
$\operatorname{Br}(\pi \pi)$ & $79.4 \%$ & $87.0 \%$ & $89.7 \%$ & $91.3 \%$ & $91.2 \%$ & $93.0 \%$ & $96.3 \%$ & $96.8 \%$ \\
$\operatorname{Br}(4 \pi)$ & $0 \%$ & $0 \%$ & $0 \%$ & $0.1 \%$ & $1.7 \%$ & $1.9 \%$ & $1.2 \%$ & $1.2 \%$ \\
\hline \hline
\end{tabular}

proportional to $\sin ^{2} \theta$. We calculate the partial decay widths for $h_{s} \rightarrow \ell^{+} \ell^{-}$in the following [15]:

$$
\Gamma\left(h_{s} \rightarrow \ell^{+} \ell^{-}\right)=\sin ^{2} \theta \frac{m_{\ell}^{2} m_{h_{s}}}{8 \pi\langle\phi\rangle^{2}}\left(1-\frac{4 m_{\ell}^{2}}{m_{h_{s}}^{2}}\right)^{3 / 2} .
$$

For our interested mass range $m_{h_{s}} \lesssim 1 \mathrm{GeV},{ }^{1}$ the light scalar almost only decays to either $\mu^{+} \mu^{-}$, a pair of pions, or four pions, depending on the phase space allowed. For $h_{s} \rightarrow \pi \pi$, a similar tree-level analytic expression given in Ref. [15] is insufficient as it fails to take into account strong final-state interactions near the pion threshold. Therefore, we adopt the following numerical treatment. We extract $\Gamma\left(h_{s} \rightarrow \pi \pi\right)$ and $\Gamma\left(h_{s} \rightarrow 4 \pi\right)$ from Ref. [18] and calculate $\Gamma\left(h_{s} \rightarrow \mu^{-} \mu^{+}\right)$with Eq. (10), in order to obtain $\Gamma\left(h_{s}\right)$, the total decay width of $h_{s}$. Then it is trivial to compute $\operatorname{Br}\left(h_{s} \rightarrow \pi \pi\right)$. Further, we calculate $\operatorname{Br}\left(h_{s} \rightarrow \pi^{+} \pi^{-}\right)$with the following formula:

$$
\operatorname{Br}\left(h_{s} \rightarrow \pi^{+} \pi^{-}\right)=\frac{2}{3} \cdot \operatorname{Br}\left(h_{s} \rightarrow \pi \pi\right),
$$

since $\Gamma\left(h_{s} \rightarrow \pi^{+} \pi^{-}\right)=2 \Gamma\left(h_{s} \rightarrow \pi^{0} \pi^{0}\right)$. In Table I, we list the decay branching ratios of $h_{s}$ for $m_{h_{s}}=0.3-1 \mathrm{GeV}$.

Before we discuss the production of $h_{s}$ from the SM Higgs decays, we present a plot of the proper decay length $c \tau_{h_{s}}$ of $h_{s}$ for $\sin ^{2} \theta=1$ as a function of its mass $m_{h_{s}}$ in Fig. 1, shown in the plane $\log _{10}\left(c \tau_{h_{s}} \cdot \sin ^{2} \theta\right)$ versus $m_{h_{s}}$.

The partial decay width of the Higgs boson into a pair of the light scalar bosons $h_{s}$ is expressed with the following analytic formula [14]:

$\Gamma\left(h \rightarrow h_{s} h_{s}\right) \simeq \frac{\langle\phi\rangle^{2}}{32 \pi m_{h}}\left(\lambda_{\Phi X}\right)^{2} \simeq \frac{\sin ^{2} \theta\left(m_{h}^{2}-m_{h_{s}}^{2}\right)^{2}}{32 \pi m_{h}\langle\chi\rangle^{2}}$,

where the first approximation makes use of the fact that the interested mass range of $h_{s}$ is negligible compared to the Higgs-boson mass and so is the phase space effect consequently, and the second approximation follows from $\sin \theta \approx \theta$ for small $\theta$ and Eq. (9). We can then calculate

\footnotetext{
${ }^{1}$ For slightly higher mass, such as $1 \mathrm{GeV} 1 \mathrm{GeV} \lesssim m_{h_{s}} \lesssim$ $2 \mathrm{GeV}$, see Ref. [16] for a search study of such scalars at the LHCb. A sensitivity study for a similar model at the LHC and HL-LHC can also be found in Ref. [17].
}

the decay branching ratio of the SM Higgs into a pair of $h_{s}$ as follows:

$$
\operatorname{Br}\left(h \rightarrow h_{s} h_{s}\right)=\frac{\Gamma\left(h \rightarrow h_{s} h_{s}\right)}{\Gamma\left(h \rightarrow h_{s} h_{s}\right)+\Gamma_{h}^{\mathrm{SM}}},
$$

where $\Gamma_{h}^{\mathrm{SM}} \simeq 4.1 \mathrm{MeV}$ for $m_{h}=125.10 \mathrm{GeV}$ [19]. It is worth mentioning that the invisible decay width measurement of the SM Higgs may constrain $\operatorname{Br}\left(h \rightarrow h_{s} h_{s}\right)$ and hence the relevant parameter space. Given the current measurement results that $\operatorname{Br}(h \rightarrow$ invisible $)<19 \%$ [20], we find that $\sin ^{2} \theta$ is bounded from above at $\sim 5.1 \times 10^{-6}$ and $\sim 5.3 \times 10^{-4}$ for $\langle\chi\rangle=10$ and $100 \mathrm{GeV}$, respectively, and is essentially independent of $m_{h_{s}}$ since the considered mass range of the light scalar is too small.

Besides the constraint from the current upper bound of the invisible decay of the Higgs boson, searches in $B$ mesons decays at fixed target, collider experiments, etc., have placed more stringent bounds on the parameter space of a Higgs-portal scalar. However, for the mass range $0.3 \mathrm{GeV}<m_{h_{s}}<1.0 \mathrm{GeV}$ considered in this work, the present limits are completely dominated by the $\mathrm{LHCb}$ search results [21,22] at $\sin ^{2} \theta \sim 10^{-7}$, which searched for $B$-meson decays into a kaon and a light scalar which further decays to a pair of muons.

\section{B. Dark glueball}

A class of "neutral-naturalness" models is proposed to solve the little hierarchy problem by predicting the existence of top partners that are singlet or only charged in the

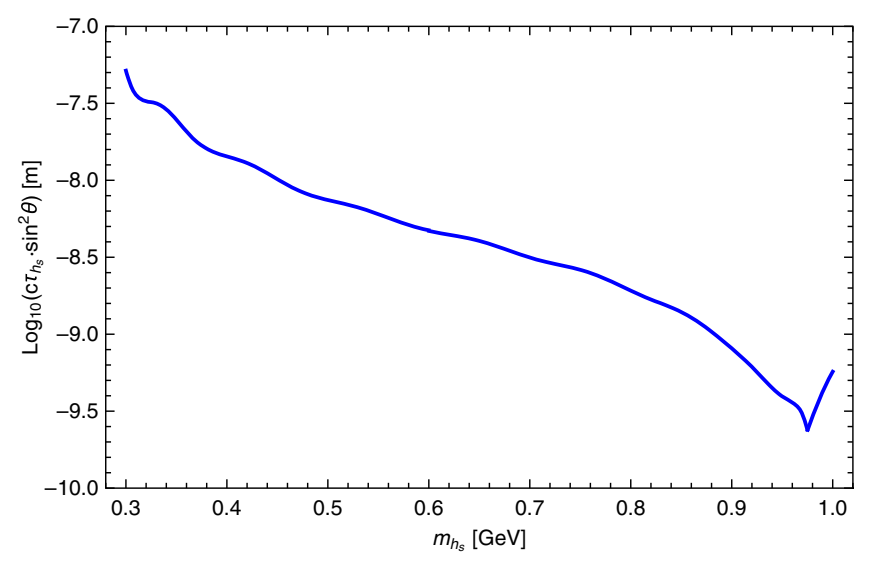

FIG. 1. $c \tau_{h_{s}} \cdot \sin ^{2} \theta$ as a function of $m_{h_{s}}$ in the mass range considered in this work. 


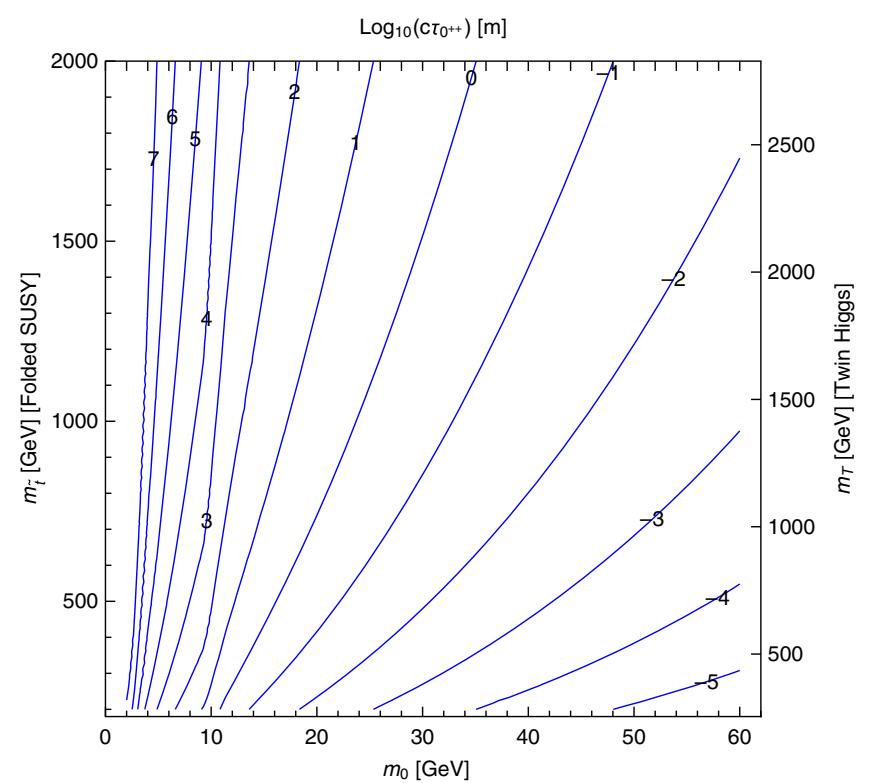

FIG. 2. The proper decay length of $0^{++}$as a function of $m_{\tilde{t} / T}$ and $m_{0}$.

SM electroweak (EW) sector, which can protect the Higgs-boson mass from large corrections at one loop up to some cutoff scales around 5-10 TeV. Such models of uncolored naturalness usually come with a dark/mirror QCD sector $S U(3)_{\mathrm{B}}$, under which the top partner is charged. In general, the mirror glueballs lie at the bottom of the mirror-sector spectrum in these models, including folded supersymmetry (SUSY) [23], (fraternal) twin Higgs [24,25], quirky little Higgs [26], and hyperbolic Higgs [27,28]. As we will see, the sensitivity reach for these models can be derived from one another by a simple rescaling, and we therefore focus on one of the models, e.g., the folded SUSY [23]. In this model, the squarks are charged under $S U(3)_{\mathrm{B}}$ (but not the $\mathrm{SM} S U(3)_{C}$ gauge group), and the EW gauge group $S U(2)_{\mathrm{L}} \times U(1)_{Y}$ is shared between the SM particles and superpartners. Since the LEP limits require that the mirror stops to be heavier than $\sim 100 \mathrm{GeV}$, the mirror glueballs are supposed to be the lightest states in the mirror sector. The lightest mirror glueball $0^{++}$can be pair produced from the Higgs-boson decay, followed by the mirrorglueball decay into a pair of SM particles via the toppartner loop-induced mixing with the SM Higgs boson, and so giving rise to displaced-vertex signatures at highenergy colliders.

For any scenario of the abovementioned models, the partial decay width of $0^{++}$into a pair of SM particles is given by [29-31]

$$
\begin{aligned}
\Gamma\left(0^{++} \rightarrow \xi \xi\right)= & \left(\frac{1}{12 \pi^{2}}\left[\frac{y^{2}}{M^{2}}\right] \frac{v}{m_{h}^{2}-m_{0}^{2}}\right)^{2} \\
& \times\left(4 \pi \alpha_{s}^{B} \mathbf{F}_{\mathbf{0}^{++}}^{\mathbf{S}}\right)^{2} \Gamma_{h \rightarrow \xi \xi}^{\mathrm{SM}}\left(m_{0}^{2}\right),
\end{aligned}
$$

where $v=246 \mathrm{GeV}$ is the SM Higgs doublet vacuum expectation value, $m_{0}$ denotes the mass of $0^{++}$, the expression for $y^{2} / M^{2}$ depends on the model and will be given in Eq. (15), and $\Gamma_{h \rightarrow \xi \xi}^{\mathrm{SM}}\left(m_{0}^{2}\right)$ is the partial decay width of a SM-like Higgs boson with mass $m_{0}$ into a pair of $\xi$ 's calculated with HDECAY $6.52 \quad[32,33] .{ }^{2} \quad 0^{++} \rightarrow \xi \xi$ here includes all the decay modes of a SM-like Higgs boson of mass $m_{0}$ into a pair of SM particles that are allowed kinematically such as $b \bar{b}$ and $\tau^{+} \tau^{-}$. For the considered mass range of $0^{++}$, i.e., $10-60 \mathrm{GeV}$, the dominant decay mode is a pair of $b$ jets. $\alpha_{s}^{B}$ and $\mathbf{F}_{\mathbf{0}^{++}}^{\mathbf{S}}$ are, respectively, the mirror strong coupling and the mirrorglueball annihilation matrix element with $4 \pi \alpha_{s}^{B} \mathbf{F}_{\mathbf{0}^{++}}^{\mathbf{S}} \approx$ $2.3 m_{0}^{3}$ [29]. The expression for $y^{2} / M^{2}$ is a compact notation for parameters in various models of neutral naturalness [30],

$$
\frac{y^{2}}{M^{2}} \approx \begin{cases}\frac{1}{4 v^{2}} \frac{m_{t}^{2}}{m_{t}^{2}}, & \text { folded SUSY, } \\ -\frac{1}{2 v^{2}} \frac{m_{t}^{2}}{m_{T}^{2}}, & \text { fraternal twin Higgs and quirky little Higgs } \\ \frac{1}{2 v^{2}} \frac{v}{v_{H}} \sin \theta, & \text { hyperbolic Higgs }\end{cases}
$$

\footnotetext{
${ }^{2}$ The current limit on the decay width of a scalar boson of mass between 10 and $-60 \mathrm{GeV}$, which mixes with the SM Higgs boson, comes from the LEP search of the SM-like Higgs boson through $h \rightarrow b \bar{b}$ and $h \rightarrow \tau \tau$ decay modes [34]. The upper bounds on the decay width of $0^{++}$are approximately $1 \%$ of a SM-like Higgs boson's decay width of the same mass $m_{0}$. Thus, the corresponding lower limits on $c \tau_{0^{++}}$are many orders of magnitude smaller than those presented in Fig. 2.
}

where $m_{t}$ is the SM top-quark mass, $m_{\tilde{t}}$ is the stop mass, $m_{T}$ is the top partner mass, and $v_{H}$ is the hyperbolic scale, and $\tan \theta \approx v / v_{H}$ encodes the tree-level mixing effects induced by the mixing between charge-parity-even neutral scalars.

As in the discussion on the Higgs-portal model, we present a plot for the proper lifetime as a function of the model parameters. In Fig. 2, we show isocurves of $\log _{10}\left(c \tau_{0^{++}}\right)$in meter in the plane $m_{\tilde{t} / T}$ versus $m_{0}$. The plot is in the same format as the corresponding plot in 
Ref. [29], but the factor- 2 correction to $y^{2} / M^{2}$ is taken here into account and leads to changes in the plot. For smaller $m_{0}$ and larger $m_{\tilde{t} / T}$, the lightest mirror glueballs have a longer proper lifetime.

As for the production of the lightest mirror glueballs from the SM Higgs decay, we calculate the relevant branching ratio as

$$
\begin{aligned}
\operatorname{Br}\left(h \rightarrow 0^{++} 0^{++}\right) \approx & \operatorname{Br}(h \rightarrow g g)_{\mathrm{SM}} \cdot\left(\frac{\alpha_{s}^{B}\left(m_{h}\right)}{\alpha_{s}^{A}\left(m_{h}\right)} 2 v^{2}\left[\frac{y^{2}}{M^{2}}\right]\right)^{2} \\
& \cdot \sqrt{1-\frac{4 m_{0}^{2}}{m_{h}^{2}}} \cdot \kappa\left(m_{0}\right),
\end{aligned}
$$

where $\operatorname{Br}(h \rightarrow g g)_{\mathrm{SM}} \approx 8.6 \%$ is the decay branching ratio of the SM Higgs boson $h$ into a pair of gluons, and $\frac{\alpha_{s}^{B}\left(m_{h}\right)}{\alpha_{s}^{A}\left(m_{h}\right)} \sim \mathcal{O}(1)$ is the ratio of the couplings of the hidden and SM QCD sectors. ${ }^{3}$ For a conservative estimate of $\alpha_{s}^{B}\left(m_{h}\right) / \alpha_{s}^{A}\left(m_{h}\right)$, we extract the information from the lower green curve of Fig. 5 of Ref. [29] for $\left(\alpha_{s}^{B}\left(m_{h}\right) / \alpha_{s}^{A}\left(m_{h}\right)\right)^{2}$, where the one-loop RGE extrapolation from $m_{0}$ was used assuming that mirror glueballs are the only mirror states below $m_{h}$. $\kappa$ is a parameter taking into account the effect of the glueball hadronization and nonperturbative mixing effects between the excited glueball states $0^{(++*)}$ and the SM Higgs boson. Following Ref. [29], the maximal value $\kappa_{\max }=1$ is the most optimistic signal estimate, while the minimal value $\kappa_{\min }$ is the most pessimistic case, which can be estimated under democratic Higgs-decay principle as follows:

$$
\kappa_{\min }\left(m_{0}\right)=\frac{\sqrt{1-\frac{4 m_{0}^{2}}{m_{h}^{2}}}}{\sum_{i} \sqrt{1-\frac{4 m_{i}^{2}}{m_{h}^{2}}}},
$$

where $i$ runs over the active states among the 12 stable glueball states, since for relatively large values of $m_{0}$, some heavier mirror-glueball states are forbidden to be produced from the Higgs decay. This is because in the mirror-glueball spectrum only the ratios $m_{i} / m_{0}$ are known [35,36].

As in the Higgs-portal model, we take into account the constraint on the parameter space from the invisible Higgs decay width measurement. We find that for $m_{\tilde{t} / T}$ as small as $100-200 \mathrm{GeV}, \operatorname{Br}\left(h \rightarrow 0^{++} 0^{++}\right)$is at the largest at $1 \%$ level, far below the present experimental upper bound 19\%. Heavier stop masses would

\footnotetext{
${ }^{3}$ Note that in Eqs. (15) and (16) there is a factor of 2 difference from the corresponding formulas given in Ref. [29]. This is because in Eq. (3.3) of Ref. [29] an overall factor 1/2 is missing compared to Eq. (2.2) of Ref. [35], the notation of which was adopted in Ref. [29].
}

give a branching ratio orders of magnitude below the current bound. Therefore, we conclude that this constraint is irrelevant for the parameter region of this model considered in this work.

\section{DETECTOR SETUPS, SIMULATION, AND CALCULATION}

By simulating 100k events for every parameter point with the Monte-Carlo simulation tool PYTHIA 8.235, we obtain the total number of signal events $N_{\text {s.e. }}$ by estimating the number of reconstructed displaced vertices in the IT, HCAL, or MS. We switch on "HiggsSM: all" of the "HiggsProcess" module in PYTHIA 8 to turn on all three Higgs production processes at an $e^{-} e^{+}$ collider, and set the SM Higgs boson to decay solely into a pair of new scalars, which decay further to a specified final state depending on which model we are studying. We place different requirements on the secondary vertices depending on the detector component and the model in order to perform the estimation. Since in each signal event there are two displaced vertices, we require at least one displaced vertex to be reconstructed in the IT in order to constitute a signal event, while for HCAL/MS we require both two vertices reconstructed inside the corresponding component. We may express $N_{\text {s.e. }}$ for the IT, HCAL, and MS with the following formulas, respectively:

$$
\begin{gathered}
N_{\text {s.e. }}^{\mathrm{IT}}=\mathcal{L}_{h} \cdot \sigma_{h} \cdot \operatorname{Br}(h \rightarrow X X) \cdot\langle P[\text { s.e. in IT }]\rangle \cdot \epsilon^{\mathrm{IT}}, \\
N_{\text {s.e. }}^{\mathrm{HCAL}}=\mathcal{L}_{h} \cdot \sigma_{h} \cdot \operatorname{Br}(h \rightarrow X X) \cdot\langle P[\text { s.e. in } \mathrm{HCAL}]\rangle, \\
N_{\text {s.e. }}^{\mathrm{MS}}=\mathcal{L}_{h} \cdot \sigma_{h} \cdot \operatorname{Br}(h \rightarrow X X) \cdot\langle P[\text { s.e. in } \mathrm{MS}]\rangle .
\end{gathered}
$$

Here $\mathcal{L}_{h}$ is the integrated luminosity at the Higgs mode, $\sigma_{h}$ is the total cross section for the SM Higgs production by combining the three processes at the $e^{-} e^{+}$colliders $\left(e^{-} e^{+} \rightarrow h Z\right.$ and two vector-boson-fusion processes: $W W$ and $Z Z$ ), $X$ represents either the light scalar boson $h_{s}$ or the lightest mirror glueball $0^{++}$, and $\epsilon^{\mathrm{IT}}$ denotes the cut efficiency for the IT. For both the CEPC and FCC-ee, the number of the Higgs bosons produced $N_{h}=\mathcal{L}_{h} \cdot \sigma_{h} \simeq 1.14 \times 10^{6}$ for operation at the Higgs mode with $\sqrt{s}=240 \mathrm{GeV}$ [12,37]. $\langle P[$ s.e. in IT $]\rangle$ denotes the average probability for at least one of the LLPs decaying inside the IT. $\langle P[$ s.e. in HCAL $]\rangle$ and $\langle P[$ s.e. in MS $]\rangle$ are similar notations employed for the HCAL and MS, respectively. These average decay probabilities may be calculated as follows: 


$$
\begin{aligned}
& \langle P[\text { s.e. in IT }]\rangle=\frac{1}{N^{\mathrm{MC}}} \sum_{i=1}^{N^{\mathrm{MC}}}\left(P\left[X_{i}^{1} \text { in IT }\right]+P\left[X_{i}^{2} \text { in IT }\right]-P\left[X_{i}^{1} \text { in IT }\right] \cdot P\left[X_{i}^{2} \text { in IT }\right]\right), \\
& \langle P[\text { s.e. in } \mathrm{HCAL}]\rangle=\frac{1}{N^{\mathrm{MC}}} \sum_{i=1}^{N^{\mathrm{MC}}}\left(P\left[X_{i}^{1} \text { in } \mathrm{HCAL}\right] \cdot P\left[X_{i}^{2} \text { in } \mathrm{HCAL}\right]\right) \text {, } \\
& \langle P[\text { s.e. in } \mathrm{MS}]\rangle=\frac{1}{N^{\mathrm{MC}}} \sum_{i=1}^{N^{\mathrm{MC}}}\left(P\left[X_{i}^{1} \text { in } \mathrm{MS}\right] \cdot P\left[X_{i}^{2} \text { in } \mathrm{MS}\right]\right),
\end{aligned}
$$

where $N^{\mathrm{MC}}$ is the total number of MC-simulated events, and $P\left[X_{i}^{1 / 2}\right.$ in IT/HCAL/MS $]$ is the individual decay probability of the first/second of the two LLPs in the $i$ th simulated signal event inside the respective fiducial component. Before we show how to calculate the latter, we first introduce the geometries of the IT, HCAL, and MS.

A profile sketch of the CEPC and FCC-ee detectors is shown in Fig. 3. The setups in the CEPC baseline detector and FCC-ee IDEA design are similar [38,39]. Summaries of the geometrical parameters used for the IT, HCAL, and MS in this work extracted from Refs. [38,39] are listed in Tables II-IV, respectively. We now describe each fiducial volume for the calculation of $P\left[X_{i}^{1 / 2}\right.$ in IT/HCAL/MS $]$. For leptonic final states $\left(h_{s} \rightarrow \mu^{-} \mu^{+}\right)$, we consider both the

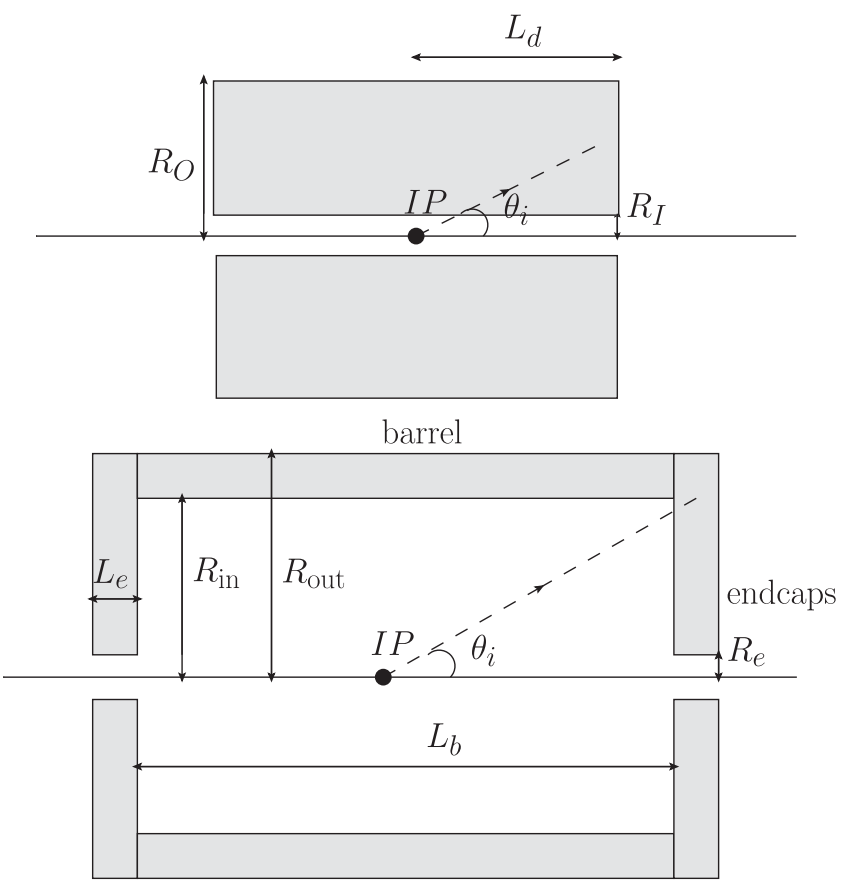

FIG. 3. Profile sketch of the detector components of the CEPC and FCC-ee. The upper plot is for the inner tracker, and the lower one is for the HCAL and muon chamber. $\theta_{i}$ labels the polar angle of an example LLP with its traveling path depicted by the accompanying dashed line with an arrow.
IT and MS for reconstructing the displaced vertices, while for the hadronic final states $\left(h_{s} \rightarrow \pi^{-} \pi^{+}\right.$and $\left.0^{++} \rightarrow b \bar{b}\right)$, we further include the HCAL as a part of the fiducial volume. For the IT, we consider the vertex detector and the silicon inner tracker. The HCAL and muon spectrometer consist of a barrel and two endcaps each.

For signal events in the IT, we require at least one displaced vertex in each event. Thus, the expression for $P\left[X_{i}\right.$ in IT $]$ is given by

$$
\begin{aligned}
P\left[X_{i} \text { in IT }\right] & =e^{-L_{i} / \lambda_{i}^{t}} \cdot\left(1-e^{-L_{i}^{\prime} / \lambda_{i}^{t}}\right), \\
L_{i} & \equiv \begin{cases}R_{I}, & \text { if }\left|L_{d} \tan \theta_{i}\right| \leq R_{I} \\
d_{\text {res }}, \text { else },\end{cases} \\
L_{i}^{\prime} & \equiv \min \left(\max \left(R_{I},\left|L_{d} \tan \theta_{i}\right|\right), R_{O}\right)-L_{i}, \\
\lambda_{i}^{t} & =\beta_{i}^{t} \gamma_{i} \tau_{X},
\end{aligned}
$$

TABLE II. Summary of parameters of the IT of the CEPC and FCC-ee IDEA. The parameters of the CEPC baseline detector are extracted from Refs. [38], while the geometries of the IDEA detectors of the FCC-ee are reproduced from Ref. [39]. $V$ represents the volume and similarly in Tables III and IV.

\begin{tabular}{|c|c|c|c|c|c|c|}
\hline Detector & $L_{b}[\mathrm{~m}]$ & $L_{e}[\mathrm{~m}]$ & $R_{e}[\mathrm{~m}]$ & $R_{\text {in }}[\mathrm{m}]$ & $R_{\text {out }}[\mathrm{m}]$ & $V\left[\mathrm{~m}^{3}\right]$ \\
\hline CEPC & 8.28 & 1.72 & 0.50 & 4.40 & 6.08 & 854.8 \\
\hline FCC-ee IDEA & 11 & 1 & 0.35 & 4.5 & 5.5 & 534.9 \\
\hline
\end{tabular}

\begin{tabular}{lcccc}
\hline \hline Detector & $R_{I}[\mathrm{~mm}]$ & $R_{O}[\mathrm{~m}]$ & $L_{d}[\mathrm{~m}]$ & $V\left[\mathrm{~m}^{3}\right]$ \\
\hline CEPC & 16 & 1.8 & 2.35 & 47.8 \\
FCC-ee IDEA & 17 & 2.0 & 2.0 & 50.3 \\
\hline \hline
\end{tabular}

TABLE III. Summary of parameters of the HCAL of the CEPC and FCC-ee IDEA.

\begin{tabular}{lcccccc}
\hline \hline Detector & $L_{b}[\mathrm{~m}]$ & $L_{e}[\mathrm{~m}]$ & $R_{e}[\mathrm{~m}]$ & $R_{\text {in }}[\mathrm{m}]$ & $R_{\text {out }}[\mathrm{m}]$ & $V\left[\mathrm{~m}^{3}\right]$ \\
\hline CEPC & 5.3 & 1.493 & 0.50 & 2.058 & 3.38 & 224.5 \\
FCC-ee IDEA & 6 & 2.5 & 0.35 & 2.5 & 4.5 & 580.1 \\
\hline \hline
\end{tabular}

TABLE IV. Summary of parameters of the MS of the CEPC and FCC-ee IDEA. 
where $\beta_{i}^{t}$ is the speed of the $X_{i}$ in the transverse direction with $\gamma_{i}$ its boost, and $\tau_{X}$ is the lifetime of $X . R_{I}\left(R_{O}\right)$ is the inner (outer) radius of the inner detector, and $L_{d}$ is its half length. $d_{\text {res }}=5 \mu \mathrm{m}$ is the inner-tracker spatial resolution for both CEPC and FCC-ee [12,37]. As long as one of the LLPs travels inside the IT window, and decays before it leaves the IT (including the case that the secondary vertex is inside the beam pipe up to $d_{\text {res }}$ ), we treat the decay vertex as a displaced vertex that can be reconstructed. The kinematical variables may be obtained with the following relations:

$$
\begin{gathered}
\beta_{i}^{t}=\left|p_{i}^{t} / E_{i}\right|, \\
\gamma_{i}=E_{i} / m,
\end{gathered}
$$

where $p_{i}^{t}, E_{i}$, and $m$ are, respectively, the transverse momentum of $X_{i}$, its energy, and its mass.

Note that for the HCAL and MS we require both displaced vertices to be reconstructed in order to render the event as a signal. The formulas of $P\left[X_{i}^{1 / 2}\right.$ in HCAL/MS $]$ for the CEPC and FCC-ee are the same, though the geometrical parameters are slightly different. The expression for $P\left[X_{i}\right.$ in $\left.\mathrm{HCAL} / \mathrm{MS}\right]$ is given by
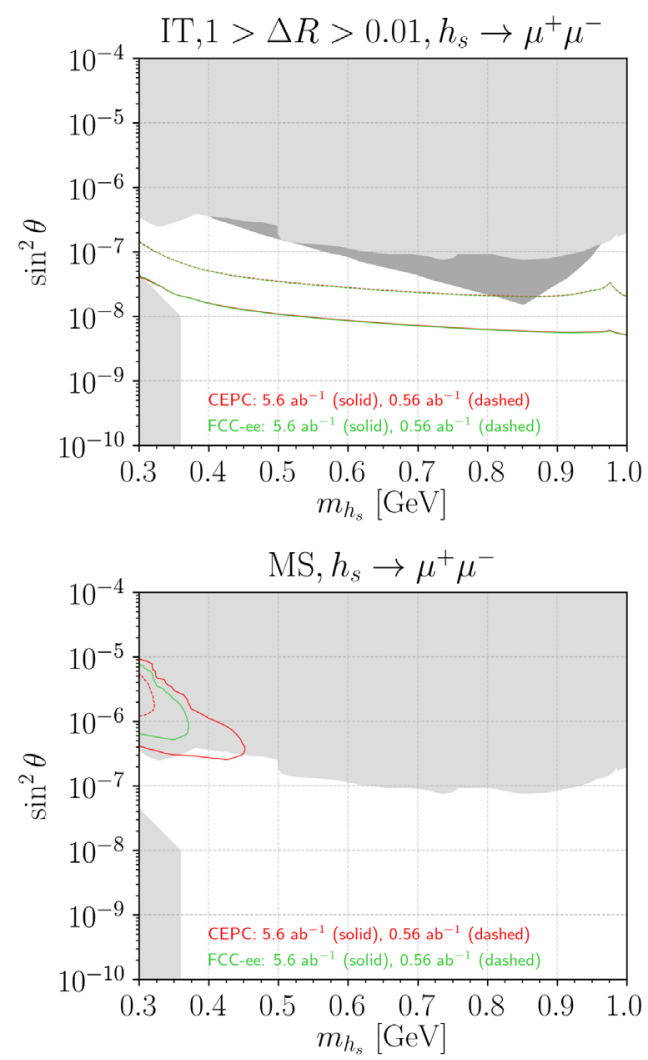

$$
\begin{aligned}
& {\left[X_{i} \text { in HCAL/MS }\right] } \\
& =e^{-R_{e} / \lambda_{i}^{z}} \cdot\left(1-e^{-L_{i}^{\alpha} / \lambda_{i}^{t}}\right)-e^{-R_{e} / \lambda_{i}^{z}} \cdot\left(1-e^{-L_{i}^{\beta} / \lambda_{i}^{t}}\right) \\
L_{i}^{\alpha} & \equiv \min \left(\max \left(R_{e},\left|\left(\frac{L_{b}}{2}+L_{e}\right) \tan \theta_{i}\right|\right), R_{\text {out }}\right)-R_{e} \\
L_{i}^{\beta} & \equiv \min \left(\max \left(R_{e},\left|\frac{L_{b}}{2} \tan \theta_{i}\right|\right), R_{\mathrm{in}}\right)-R_{e}
\end{aligned}
$$

where $R_{\text {in }}\left(R_{\text {out }}\right)$ is the inner (outer) radius of the barrel, $L_{b}$ its full length, and $L_{e}\left(R_{e}\right)$ is the width (inner radius) of the two endcaps.

\section{RESULTS}

In this section, we present the numerical results of the sensitivity reach of the CEPC and FCC-ee for the two models considered in this study.

\section{A. The light sub-GeV scalar boson case}

We present the sensitivity estimates in the plane $\left(\sin ^{2} \theta\right.$ vs $m_{h_{s}}$ ) for the light sub-GeV scalar-boson model in Figs. 4 and 5 for the channels $h_{s} \rightarrow \mu^{-} \mu^{+}$and $h_{s} \rightarrow$ $\pi^{-} \pi^{+}$, respectively. We consider the number of signal events larger or equal to 3 as the sensitive region, which corresponds to the exclusion limit of 95\% C.L (confidence
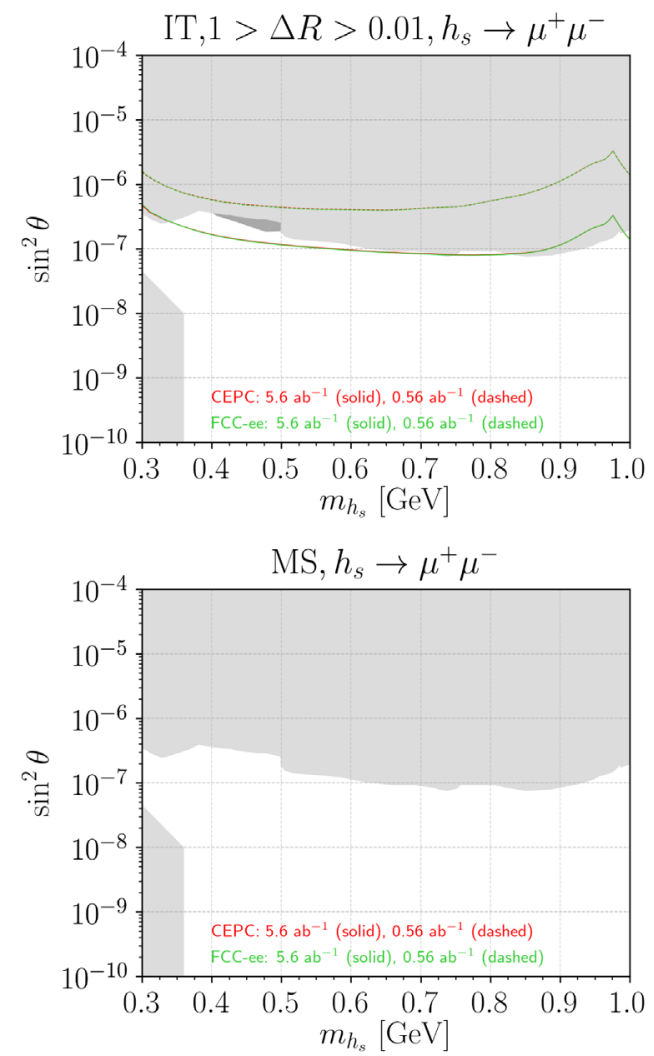

FIG. 4. Sensitivity reaches at the CEPC and FCC-ee for $h_{s} \rightarrow \mu^{+} \mu^{-}$. The left panels correspond to $\langle\chi\rangle=10 \mathrm{GeV}$, while the right ones to $\langle\chi\rangle=100 \mathrm{GeV}$. The light gray area is experimentally excluded, while the dark gray part shown in the upper row can be probed at the LHC with $300 \mathrm{fb}^{-1}$ integrated luminosity. 

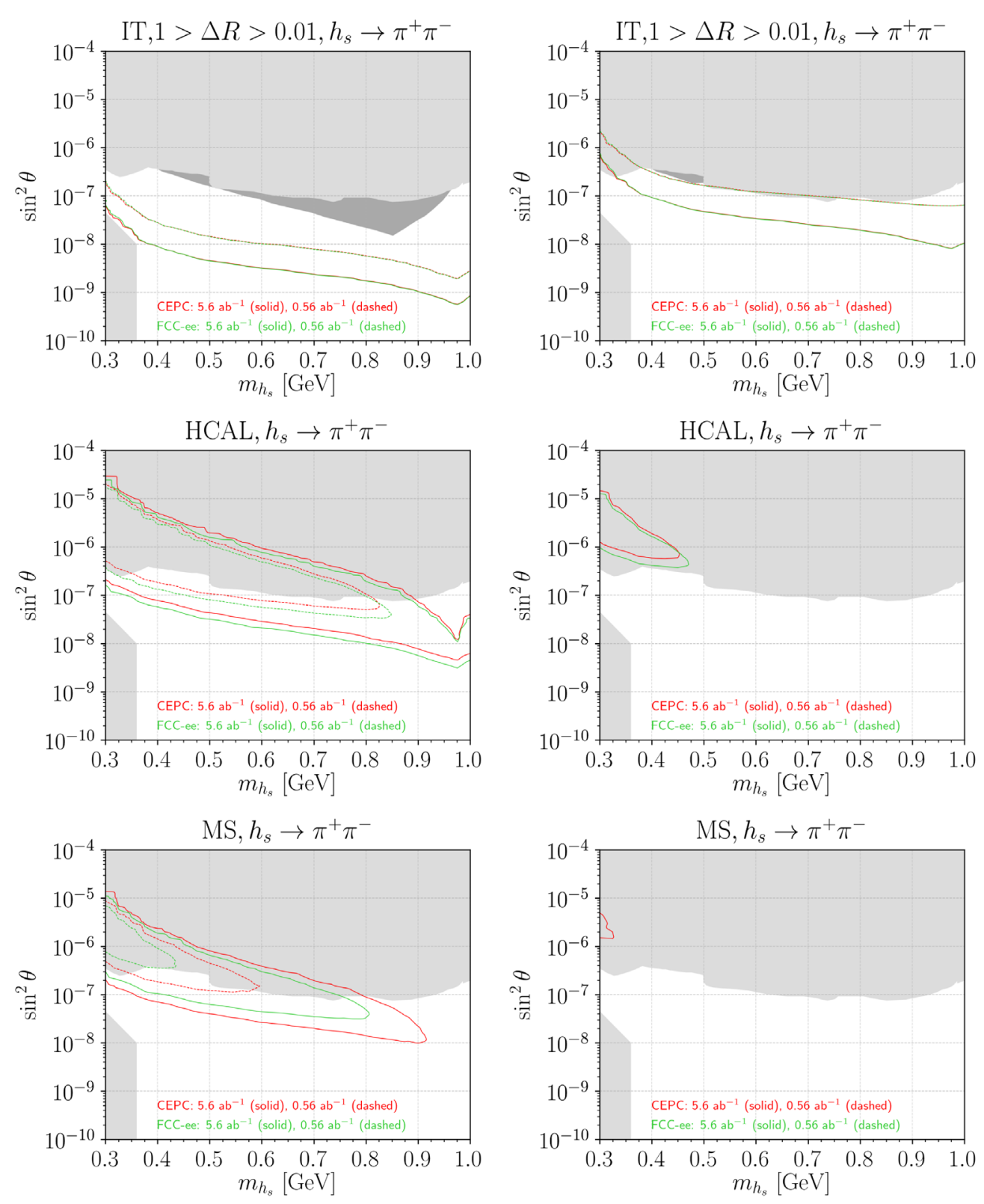

FIG. 5. Sensitivity reaches at the CEPC and FCC-ee for $h_{s} \rightarrow \pi^{+} \pi^{-}$. The left panels correspond to $\langle\chi\rangle=10 \mathrm{GeV}$, while the right ones to $\langle\chi\rangle=100 \mathrm{GeV}$. The light gray area is experimentally excluded, while the dark gray part shown in the upper row can be probed at the LHC with $300 \mathrm{fb}^{-1}$ integrated luminosity.

level). with zero background event. The ITs of the CEPC and FCC-ee employ similar geometries and consequently their results are almost identical, while the HCAL/MS of the two detectors somewhat differ in their dimensions and hence volumes, leading to slightly different results. In Figs. 4 and 5, the solid lines represent the projected integrated luminosity of $\mathcal{L}_{h}=5.6 \mathrm{ab}^{-1}$, while the dashed ones are for $\mathcal{L}_{h}=0.56 \mathrm{ab}^{-1}$ only. We show results for these two integrated luminosities in order to achieve fair comparisons with the results in Ref. [14], where the LHC sensitivities for the same model with dimuon displaced vertices were obtained for an integrated luminosity of
$300 \mathrm{fb}^{-1}$ while at the end of high-luminosity LHC (HLLHC) run $3 \mathrm{ab}^{-1}$ of data is expected. We apply a selection cut on the opening angle of the two muons/pions produced from the $h_{s}$ decays inside the IT: $1>\Delta R>0.01$. This can effectively eliminate the contributions from background events of heavier particles while respecting the tracking spatial resolution. As for the HCAL and MS, we assume zero background events. The LHC results from Ref. [14] are reproduced here in Figs. 4 and 5 for easy comparisons. The two benchmark values of $\langle\chi\rangle=10,100 \mathrm{GeV}$ are chosen as we consider light $h_{s}$ of sub-GeV mass. The light gray area is the experimentally excluded region by 

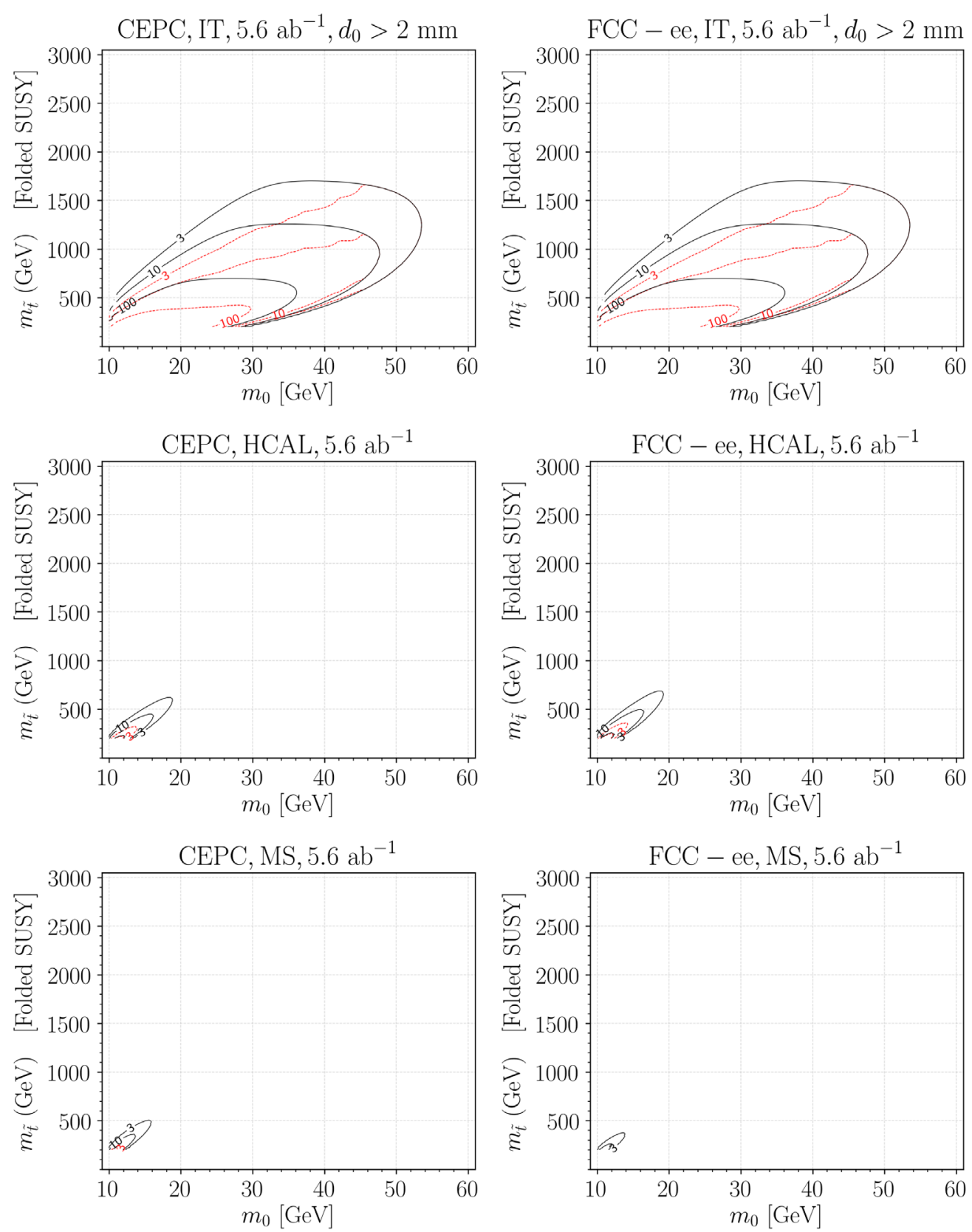

FIG. 6. Sensitivity reaches of $\log _{10}\left(N_{\text {signal }}\right)$ at the CEPC and FCC-ee for the folded SUSY model. The black(red) curves correspond to $\kappa=\kappa_{\max }\left(\kappa=\kappa_{\min }\right)$.

fixed-target experiments, $\mathrm{LHCb}$, and B-factories [14,21, 22,40], while the dark gray area is the experimentally allowed and LHC-sensitive region [14]. Note that the strongest limits for $m_{h_{s}}$ between 0.3 and $1.0 \mathrm{GeV}$ were obtained by the LHCb searches [21,22] for $B$-meson decays. Therefore, we refrain from presenting a comprehensive overview of the previous searches which had weaker limits for the relevant mass range. See the scalar-portal discussion in Ref. [41] for a summary of the upto-date experimental constraints for $m_{h_{s}} \lesssim 10 \mathrm{GeV}$.

For the dimuon signature case, with the smaller $\langle\chi\rangle=10 \mathrm{GeV}$, the CEPC and FCC-ee with an integrated luminosity of $0.56 \mathrm{ab}^{-1}$ are slightly weaker at $\sim m_{h_{\mathrm{s}}}=$ $0.85 \mathrm{GeV}$, but can have as much as a 2 orders of magnitude advantage in the rest of the considered $m_{h_{s}}$ range. As for $\langle\chi\rangle=100 \mathrm{GeV}$, the performance of the $e^{-} e^{+}$colliders with an integrated luminosity of $0.56 \mathrm{ab}^{-1}$ is inferior to the LHC for most of the mass range. Furthermore, with the integrated luminosity of $5.6 \mathrm{ab}^{-1}$, the performance is better than the LHC with $300 \mathrm{fb}^{-1}$ integrated luminosity for both small and large $\langle\chi\rangle$ values. While the sensitivity reach of the IT is dominant, our MS estimates still show some potential in the parameter space, at least in the $\langle\chi\rangle=$ $10 \mathrm{GeV}$ case, though for $\langle\chi\rangle=100 \mathrm{GeV}$ the MS has no 
sensitivity in the considered mass range as there would be too few $h_{s}$ produced.

On the other hand, by including the signatures from displaced charged pions, one may significantly enhance the signal sensitivities, as for $m_{h_{s}} \lesssim 1 \mathrm{GeV}$ the light scalar boson almost solely decays into (neutral or charged) pions. We expect that collimated pions can be detected thanks to the clean environment at $e^{-} e^{+}$colliders. The results are shown in Fig. 5. Roughly, for $m_{h_{s}} \gtrsim 0.7 \mathrm{GeV}$, we observe that the sensitivities improve by orders of magnitude compared to those in the dimuon signature case. Now, the CEPC/FCC-ee IT may explore $\sin ^{2} \theta$ a few orders of magnitude lower than the sensitive region of the LHC for the whole mass range. In fact, for $\langle\chi\rangle=10 \mathrm{GeV}$, even the HCAL with $\mathcal{L}=0.56 \mathrm{ab}^{-1}$ may have a comparable reach to that of the LHC. Similar to the dimuon signature, for $\langle\chi\rangle=100 \mathrm{GeV}$, the sensitivities at the HCAL and MS are worsened by a large extent because of the reduced production cross section of $h_{s}$.

\section{B. The mirror-glueball case}

We now present the results for the mirror-glueball case. We focus on their decay into a pair of $b$ jets as this channel has the largest decay branching ratio for $0^{++}$in the mass range under consideration, and consequently we perform
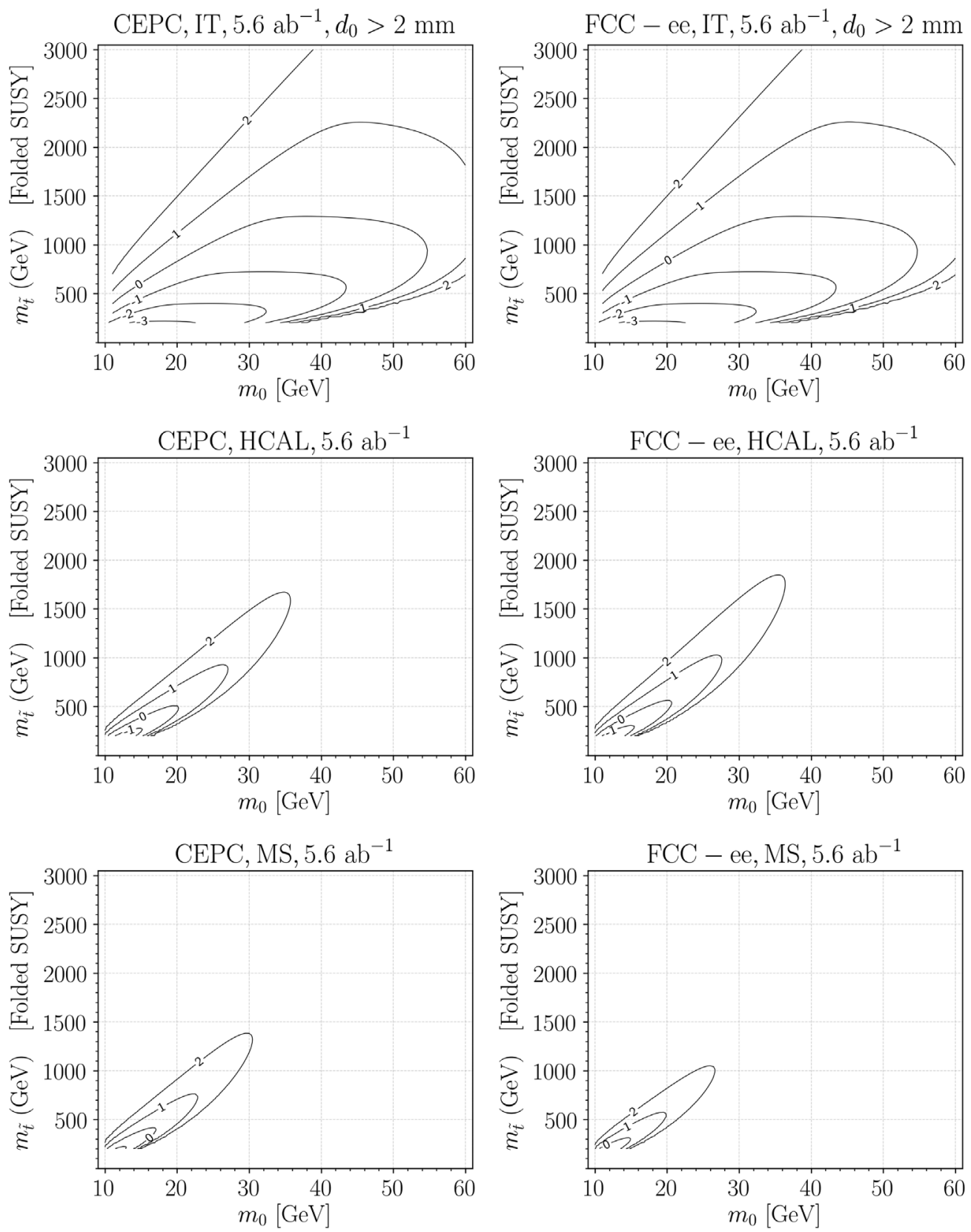

FIG. 7. Sensitivity reaches of $\log _{10}(\kappa)$ at the CEPC and FCC-ee for the folded SUSY model for $N_{\text {signal }}=10$. 
sensitivity estimation for all of the IT, HCAL, and MS. There are two major backgrounds under our consideration, i.e., $e^{-} e^{+} \rightarrow Z Z \rightarrow\left(\ell^{+} \ell^{-}, j j\right)(b \bar{b})$ and $e^{-} e^{+} \rightarrow Z h \rightarrow$ $\left(\ell^{+} \ell^{-}, j j\right)(b \bar{b})$, in which the $b \bar{b}$ pair comes from prompt $Z$ or $h$ decay. We make use of all visible decays $\left(\ell^{+} \ell^{-}, q \bar{q}\right)$ of the $Z$ boson, which corresponds to a branching ratio of $\sim 0.8$. An invariant mass cut is imposed on the lepton pair or quark pair to identify the $Z$ boson. It is followed by a recoilmass cut, defined by

$$
\begin{aligned}
M_{\text {recoil }}^{2} & =s-2 \sqrt{s}\left(E_{\ell^{+}}+E_{\ell^{-}}\right)+M_{\ell \ell}^{2} ; \\
120 \mathrm{GeV} & <M_{\text {recoil }}<150 \mathrm{GeV},
\end{aligned}
$$

in order to remove the $Z Z$ background. It was shown in Ref. [25] that such a cut can reduce the $Z Z$ background down to less than $10^{-2}$. In order to remove the prompt $h / Z \rightarrow b \bar{b}$ decay and the SM bottomonium background events of displaced vertices taking place in the IT, we further impose an invariant-mass cut on the $b \bar{b}$ pair $10 \mathrm{GeV}<M_{b \bar{b}}<80 \mathrm{GeV}$, which has no effect on the signal events. Furthermore, we require the transverse impact parameter $d_{0}>2 \mathrm{~mm}$ for both $b$ jets stemming from any secondary vertex in the IT, so as to make sure that the corresponding vertex is a displaced one and can be reconstructed. This would cut away some sensitivity at the ultrashort decay length regime (corresponding to the lower right corner in the shown plane $m_{\tilde{t}}$ vs $m_{0}$ ). Similar to the previous model, we have assumed that for the HCAL and MS the SM background is negligible.

We present our sensitivity estimates in two ways. In Fig. 6, we make plots of contour curves for $N_{\text {signal }}$ denoting the number of signal events. The selected isocurves are for $N_{\text {signal }}=3,10,100$. The left and right columns correspond to the CEPC and FCC-ee, respectively. The black (red) curves are for $\kappa_{\max }\left(\kappa_{\min }\right)$. In Ref. [30], the limits for the same model were also shown for the CEPC/FCC-ee with $N_{h}=1.1 \times 10^{6}$. Compared to the results therein, our estimates for the IT (with three signal events or 95\% C.L. with zero background) are more optimistic with the maximal potential reach of $m_{\tilde{t}}$ roughly two times better. This is largely due to the fact that we include both $l^{-} l^{+}$and $j j$ decay channels of the $Z$ bosons while Ref. [30] considered only leptonic $Z$ decays. Our sensitivity reach in $m_{0}$ is however smaller, because the requirement on the transverse impact parameter cuts away most signal events for large decay width. Compared to the IT, the HCAL and MS may have smaller sensitivity but still useful coverage in the parameter space.

In Fig. 7, we present another set of plots with contour curves for $\log _{10}(\kappa)$. The selected values of $\log _{10}(\kappa)=$ $-3,-2,-1,0,1,2$. We may compare our results with those for the HL-LHC with $\sqrt{s}=14 \mathrm{TeV}$ and $3 \mathrm{ab}^{-1}$ integrated luminosity presented in Ref. [29]. Our IT search may probe $m_{\tilde{t}}$ roughly 1.5 times better while our HCAL and MS limits are similar or slightly worse.
Note that we only show our results for the folded SUSY model. For the other neutral-naturalness models, as the parameter $y^{2} / M^{2}$ is also inversely proportional to the square of the new scale, one can easily obtain the corresponding sensitivity estimates by simple rescaling.

\section{CONCLUSIONS}

We have investigated the potential of the future $e^{-} e^{+}$ colliders operated as the Higgs factories, with the profiles of CEPC and FCC-ee as examples, in detecting the longlived particles predicted by a number of models beyond the SM. We have employed two representative models, i.e., the hidden scalar model and folded SUSY model, which feature two distinct mass ranges, including sub-GeV and $O(10) \mathrm{GeV}$, respectively.

The decay of the sub-GeV scalar boson gives rise to a pair of collimated muons or pions, which provides a distinctive signature against possible SM backgrounds. As a result of a much larger decay branching ratio into the pion pair, the sensitivity reach at CEPC and FCC-ee can be substantially better than the LHC.

The decay of relatively heavier mirror glueballs of mass $O(10) \mathrm{GeV}$ leads to a pair of $b$ jets with a clean secondary vertex. With a series of selection cuts, all possible SM backgrounds can be rejected while the signal events remain largely unaffected. By including both the leptonic and hadronic decays of the $Z$ bosons, the sensitivity reach at the CEPC and FCC-ee is about a few times better than previous studies.

We offer a few more comments as follows.

(1) In this study, we have assumed that with the selection cuts, such as collimated muon or pion pairs for the sub-GeV scalar boson and $b$-jet pairs with a secondary vertex and large invariant mass for the folded SUSY model, most SM backgrounds can be eliminated.

(2) In principle, one can also study the sensitivity reach for the ILC with the proposed geometries. However, the designed luminosity is relatively low so that one expects only fewer than 400k Higgs bosons to be produced at $\sqrt{s}=250$ or $500 \mathrm{GeV}$ after a luminosity upgrade [42], which is still below the expectation at the CEPC/FCC-ee.

(3) Another interesting mass range $O(1-10) \mathrm{GeV}$ is worth studying, because the decay of such a LLP may suffer from the SM backgrounds of $B$ hadrons.

(4) The folded SUSY model is just an example of the neutral-naturalness models. Results for the other similar models can be easily obtained by rescaling the parameter $y^{2} / M^{2}$.

\section{ACKNOWLEDGMENTS}

We thank Nathaniel Craig, Chih-Ting Lu, Yue-Lin Sming Tsai, Michael Williams, and José Francisco Zurita for useful discussions. Z. S. W. is supported by 
the Ministry of Science, ICT and Future Planning of Korea, the Pohang City Government, and the Gyeongsangbuk-do Provincial Government through the Young Scientist Training Asia-Pacific Economic Cooperation program of
APCTP, and thanks the National Center for Theoretical Sciences for hospitality where part of this work was conducted. K. C. is supported by the MoST of Taiwan under Grant No. 107-2112-M-007-029-MY3.
[1] G. Aad et al. (ATLAS Collaboration), Observation of a new particle in the search for the Standard Model Higgs boson with the ATLAS detector at the LHC, Phys. Lett. B 716, 1 (2012).

[2] S. Chatrchyan et al. (CMS Collaboration), Observation of a new boson at a mass of $125 \mathrm{GeV}$ with the CMS experiment at the LHC, Phys. Lett. B 716, 30 (2012).

[3] J. Alimena et al., Searching for long-lived particles beyond the Standard Model at the Large Hadron Collider, arXiv:1903.04497.

[4] G. Aad et al. (ATLAS Collaboration), Search for light longlived neutral particles produced in $p p$ collisions at $\sqrt{s}=$ $13 \mathrm{TeV}$ and decaying into collimated leptons or light hadrons with the ATLAS detector, arXiv:1909.01246.

[5] G. Aad et al. (ATLAS Collaboration), Search for displaced vertices of oppositely charged leptons from decays of longlived particles in $p p$ collisions at $\sqrt{s}=13 \mathrm{TeV}$ with the ATLAS detector, Phys. Lett. B 801, 135114 (2020).

[6] M. Aaboud et al. (ATLAS Collaboration), Search for longlived neutral particles in $p p$ collisions at $\sqrt{s}=13 \mathrm{TeV}$ that decay into displaced hadronic jets in the ATLAS calorimeter, Eur. Phys. J. C 79, 481 (2019).

[7] M. Aaboud et al. (ATLAS Collaboration), Search for longlived particles in final states with displaced dimuon vertices in $p p$ collisions at $\sqrt{s}=13 \mathrm{TeV}$ with the ATLAS detector, Phys. Rev. D 99, 012001 (2019).

[8] A. M. Sirunyan et al. (CMS Collaboration), Search for longlived particles decaying into displaced jets in proton-proton collisions at $\sqrt{s}=13 \mathrm{TeV}$, Phys. Rev. D 99, 032011 (2019).

[9] G. Aad et al. (ATLAS Collaboration), Triggers for displaced decays of long-lived neutral particles in the ATLAS detector, J. Instrum. 8, P07015 (2013).

[10] CMS Collaboration, First level track jet trigger for displaced jets at high luminosity LHC, CERN Report No. CMS-PASFTR-18-018, 2020.

[11] M. Ahmad et al., CEPC-SPPC preliminary conceptual design report. 1. Physics and detector, Reports No. IHEPCEPC-DR-2015-01, IHEP-TH-2015-01, IHEP-EP-2015$01,2015$.

[12] A. Abada et al. (FCC Collaboration), FCC-ee: The Lepton Collider, Eur. Phys. J. Spec. Top. 228, 261 (2019).

[13] G. Aarons et al. (ILC Collaboration), International linear collider reference design report volume 2: Physics at the ILC, arXiv:0709.1893.

[14] J. Chang, K. Cheung, S.-C. Hsu, and C.-T. Lu, Detecting multimuon jets from the Higgs boson exotic decays in the Higgs portal framework, Phys. Rev. D 95, 035012 (2017).
[15] J. F. Gunion, H. E. Haber, G. L. Kane, and S. Dawson, The Higgs Hunter's guide, Front. Phys. 80, 1 (2000).

[16] X. Cid Vidal, Y. Tsai, and J. Zurita, Identifying exclusive displaced hadronic signatures in the forward region of the LHC, arXiv:1910.05225.

[17] S. Matsumoto, Y.-L.S. Tsai, and P.-Y. Tseng, Light fermionic WIMP dark matter with light scalar mediator, J. High Energy Phys. 07 (2019) 050.

[18] M. W. Winkler, Decay and detection of a light scalar boson mixing with the Higgs boson, Phys. Rev. D 99, 015018 (2019).

[19] CERN Yellow Report, https://twiki.cern.ch/twiki/bin/view/ LHCPhysics/CERNYellowReportPageBR.

[20] A. M. Sirunyan et al. (CMS Collaboration), Search for invisible decays of a Higgs boson produced through vector boson fusion in proton-proton collisions at $\sqrt{s}=13 \mathrm{TeV}$, Phys. Lett. B 793, 520 (2019).

[21] R. Aaij et al. (LHCb Collaboration), Search for hiddensector bosons in $B^{0} \rightarrow K^{* 0} \mu^{+} \mu^{-}$decays, Phys. Rev. Lett. 115, 161802 (2015).

[22] R. Aaij et al. (LHCb Collaboration), Search for long-lived scalar particles in $B^{+} \rightarrow K^{+} \chi\left(\mu^{+} \mu^{-}\right)$decays, Phys. Rev. D 95, 071101 (2017).

[23] G. Burdman, Z. Chacko, H.-S. Goh, and R. Harnik, Folded supersymmetry and the LEP paradox, J. High Energy Phys. 02 (2007) 009.

[24] Z. Chacko, H.-S. Goh, and R. Harnik, The Twin Higgs: Natural electroweak breaking from mirror symmetry, Phys. Rev. Lett. 96, 231802 (2006).

[25] N. Craig, A. Katz, M. Strassler, and R. Sundrum, Naturalness in the dark at the LHC, J. High Energy Phys. 07 (2015) 105.

[26] H. Cai, H.-C. Cheng, and J. Terning, A quirky little Higgs model, J. High Energy Phys. 05 (2009) 045.

[27] T. Cohen, N. Craig, G. F. Giudice, and M. Mccullough, The hyperbolic Higgs, J. High Energy Phys. 05 (2018) 091.

[28] H.-C. Cheng, L. Li, E. Salvioni, and C. B. Verhaaren, Singlet scalar top partners from accidental supersymmetry, J. High Energy Phys. 05 (2018) 057.

[29] D. Curtin and C. B. Verhaaren, Discovering uncolored naturalness in exotic Higgs decays, J. High Energy Phys. 12 (2015) 072.

[30] S. Alipour-Fard, N. Craig, M. Jiang, and S. Koren, Long live the Higgs factory: Higgs decays to long-lived particles at future Lepton Colliders, Chin. Phys. C 43, 053101 (2019).

[31] A. Ahmed, Heavy Higgs of the twin Higgs models, J. High Energy Phys. 02 (2018) 048.

[32] A. Djouadi, J. Kalinowski, and M. Spira, HDECAY: A program for Higgs boson decays in the standard model and 
its supersymmetric extension, Comput. Phys. Commun. 108, 56 (1998).

[33] A. Djouadi, J. Kalinowski, M. Muehlleitner, and M. Spira, HDECAY: Twenty ${ }_{++}$years after, Comput. Phys. Commun. 238, 214 (2019).

[34] R. Barate et al. (ALEPH, DELPHI, L3, OPAL Collaborations and LEP Working Group for Higgs Boson searches), Phys. Lett. B 565, 61 (2003).

[35] J. E. Juknevich, Pure-glue hidden valleys through the Higgs portal, J. High Energy Phys. 08 (2010) 121.

[36] C. J. Morningstar and M. J. Peardon, The glueball spectrum from an anisotropic lattice study, Phys. Rev. D 60, 034509 (1999).

[37] CEPC Study Group, CEPC conceptual design report: Volume 1-accelerator, arXiv:1809.00285.
[38] M. Dong et al. (CEPC Study Group), CEPC conceptual design report: Volume 2-physics and detector, arXiv: 1811.10545.

[39] A. Abada et al. (FCC Collaboration), Future circular collider, Eur. Phys. J. ST 228, 261 (2019).

[40] J. D. Clarke, R. Foot, and R. R. Volkas, Phenomenology of a very light scalar $\left(100 \mathrm{MeV}<m_{h}<10 \mathrm{GeV}\right)$ mixing with the SM Higgs, J. High Energy Phys. 02 (2014) 123.

[41] J. Beacham et al., Physics beyond colliders at CERN: beyond the Standard Model working group report, J. Phys. G 47, 010501 (2020).

[42] F. Borzumati and E. Kato, The Higgs boson and the international linear collider, Front. Phys. 2, 32 (2014). 